International Journal of Modern Physics C, Vol. 0, No. 0 (0000) 000-000

(C) World Scientific Publishing Company

\title{
ORIENTATION CORRELATION IN SIMPLIFIED MODELS OF POLYMER MELTS
}

ROLAND FALLER, MATHIAS PÜTZ and FLORIAN MÜLLER-PLATHE

Max-Planck-Institut für Polymerforschung,

Ackermannweg 10, D-55128 Mainz, Germany

Received

Revised

\begin{abstract}
We investigate mutual local chain order in systems of fully flexible polymer melts in a simple generic bead-spring model. The excluded-volume interaction together with the connectivity leads to local ordering effects which are independent of chain length between 25 and 700 monomers, i.e. in the Rouse as well as in the reptation regime. These ordering phenomena extend to a distance of about 3 to 4 monomer sizes and decay to zero afterwards.
\end{abstract}

Keywords: molecular dynamics, orientation correlation, polymer melts

\section{Introduction}

Nuclear magnetic resonance (NMR) experiments on polymer melts and glasses show effects of mutual local ordering of neighboring chains ${ }^{1,2}$. These effects are found far away from the glass transition in non liquid-crystalline polymer melts like polybutadiene in the reptation regime. Ordering effects in polymer melts were already investigated in several molecular dynamics simulations of specific polymer melts ${ }^{3,4,5}$, block copolymers ${ }^{6}$ and by Monte Carlo methods using lattice models ${ }^{7}$. However, to date, only very few investigations of local order in small systems were performed $^{8}$ in generic off-lattice models, although these models are rather well investigated in other contexts ${ }^{9}$. It is not clear how much influence depends on the specific polymer interactions and how much orientational correlation is contributed by the excluded volume interaction which may be modeled regardless of the specific chemical system. In this work, we focus on the generic effects of local order in fully flexible polymer melts. 


\section{Method}

We performed molecular dynamics simulation of fully flexible polymer chains using a truncated and shifted Lennard-Jones potential (Weeks-Chandler-Anderson potential) for the excluded volume interaction between all beads

$$
V_{L J}(r)=4 \epsilon\left[\left(\frac{\sigma}{r}\right)^{12}-\left(\frac{\sigma}{r}\right)^{6}\right], r=r_{c}=\sqrt[6]{2} \sigma
$$

and a finitely extendable non-linear elastic (FENE) potential

$$
V_{F E N E}(r)=\frac{\alpha}{2} \frac{R^{2}}{\sigma^{2}} \ln \left(1-\frac{r^{2}}{R^{2}}\right), r<R=1.5 \sigma, \alpha=30 \epsilon
$$

to model the connectivity along the chains. The system uses orthorombic periodic boundary conditions. Temperature is kept constant by simulation of a Langevin equation with a stochastic force and a friction term added to Newton's equation of motion. The simulations were performed at a number density of $\rho=0.85$ in reduced units and a temperature of $T=1.0$ with a friction strength of $\xi=0.5$. The program used is described in detail in reference ${ }^{10}$. Our melt simulations consist of chains with 25 to 700 monomers. The overall number of monomers in the simulation lies between 80.000 and 500.000 .

We investigated the correlation between unit vectors defined along the chain

$$
\vec{u}_{d}=\overrightarrow{r_{i}}-\vec{r}_{i-d} .
$$

A value of $d=1$ means therefore just bond vectors between connected beads. The static correlation of these unit vectors is calculated. The 2 nd Legendre polynomial of the scalar product

$$
P_{2}(r)=\frac{1}{2}\left\langle 3(\vec{u}(0) \cdot \vec{u}(r))^{2}-1\right\rangle
$$

was recorded versus the distance of the center of mass of the two chain segments and averaged over all corresponding pairs with equal distance. We chose this orientational distribution function (ODF) because it is the relevant quantity in NMR measurements and it makes no sense to distinguish between the two possible directions of the chains. The orientational distribution function is negative when the two vectors are perpendicular and positive for parallel orientation. If the vectors are uncorrelated the function is zero. In our investigations, we only looked at the inter-chain effects. All correlations along one chain were explicitly excluded.

\section{Results and Discussion}

The orientational distribution functions of chains at length 25 and 700 are shown in figure 1 on the left hand side for $d=1$. The entanglement length of this system was earlier found to be 35 beads ${ }^{9}$ whereas recent simulations are more consistent with 60. The simulation with 25 and 50 monomers is in the Rouse regime, simulations with 100 or 200 monomers are in the region of crossover, whereas the longest simulations (700 monomers) are in the reptation regime. The structure and the numerical 
values of the peaks are almost indistinguishable between different chain lengths. This shows that the ordering phenomenon is strictly local and is probably imposed by chain packing. The initial negative region indicates nearly perfect perpendicular orientation. This may be understood if one realizes that the bond center distance is clearly smaller than the distance of the first peak in the radial distribution function $(\sqrt[6]{2})$. Hence only a perpendicular alignment is compatible with this small distance. Such close contacts, however, are extremely rare. The radial distribution function here is almost zero. The first positive peak in the ODF coincides rather well with the first peak in the interchain radial distribution function (figure 1 right hand side, solid line). The depression of $g(r)_{\text {inter }}$ at low distances is due to the enhanced density of the same chain in the vicinity of a monomer (correlation hole).

At the distance of the first neighbor shell, the monomers are preferentially parallel oriented. This is consistent with results on short chains of only 10 or 20 monomers ${ }^{8}$. The values of $P_{2}$ of individual pairs of vectors vary in a wide range between strongly negative and strongly positive values. Only a slight preference of the parallel orientation leads to this peak. This preferred alignment is, in general, not mediated by an intermediate perpendicular chain.
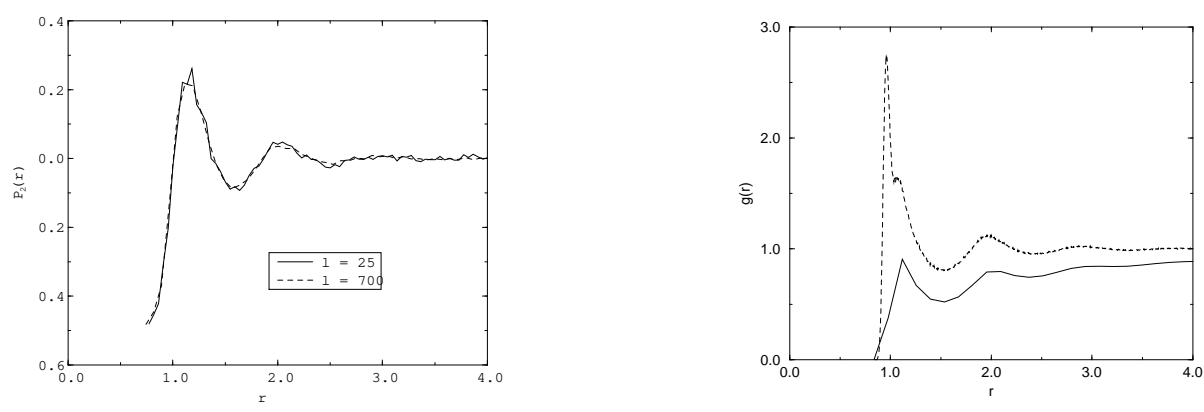

Fig. 1. left: Orientational distribution functions of chains with 25 (3200 chains), 50 (5000 chains), 200 (2500 chains ) and 700 (500 chains) monomers for vectors connecting next neighbors. right: Radial distribution functions $g(r)$ of a melt with 2500 chains of 200 monomers. Dashed line: overall $g(r)$, solid line: interchain $g(r)$.

Orientational correlations are visible up to a distance of about 3 monomer radii. The ODF falls to zero at longer distances, showing that there is no nematic longrange ordering which of course is not expected in fully flexible systems. Figure 2 shows typical local arrangements which may lead to the observed orientational distribution functions. 

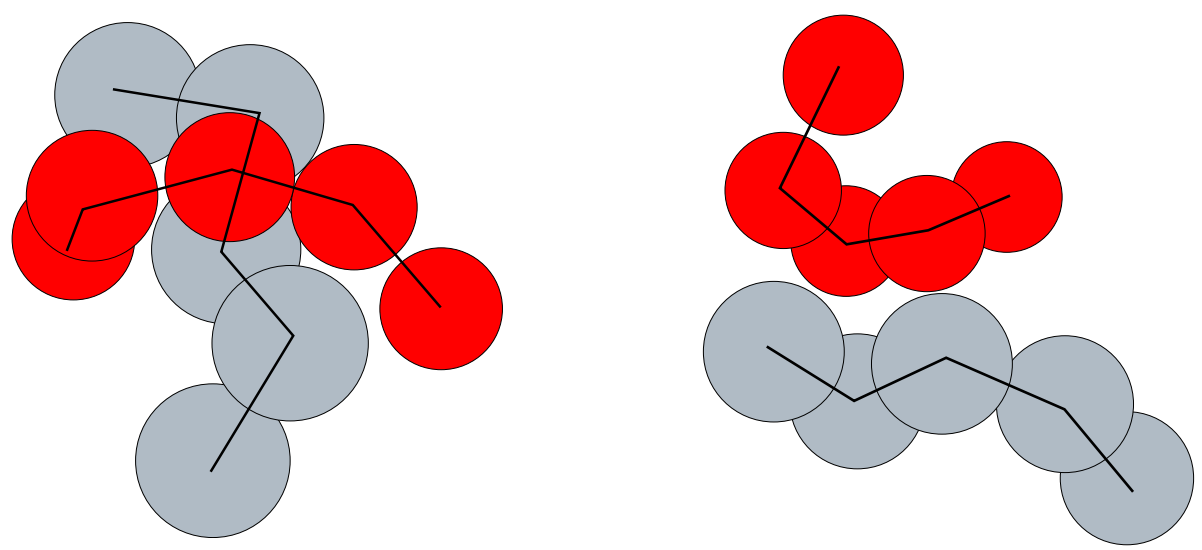

Fig. 2. Selected arrangements leading to negative (left) or positive (right) orientational distribution functions.

We also investigated the ordering of longer chain segments. We defined unit vectors in the direction from one monomer to its next-nearest neighbor $(d=2)$, next to next-nearest $(d=3)$ etc. The orientational correlations are weaker but remain visible (see figure 3 ).
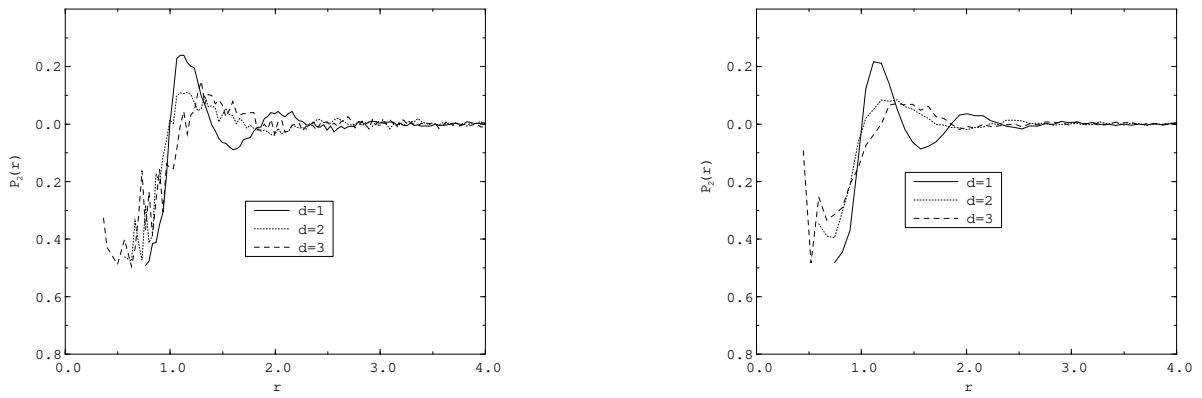

Fig. 3. Orientational distribution functions of chains with 50 monomers (5000 chains,left) and 700 monomers (500 chains, right) depending on segment length $d$ (see text)

The first peak is shifted to longer distances which is not surprising because of the bigger segment size. This shows that the local ordering of the bonds also leads to an ordering of segments of several beads. The maximum of the ODF for $l=700$ and $d=2,3$ seems to be at a slightly bigger distance, but this is not yet significant. The orientational distribution functions are still too noisy to decide this clearly.

To summarize, we find local ordering of adjacent polymer chains in the melt even for a fully flexible model with no interactions other than excluded volume. This ordering does not depend on the overall chain length since it is a local property. Even the otherwise drastic difference between entangled and unentangled systems does 
not influence the static correlations. Presumably, the lifetime of the correlations is shorter than the time-scale relevant for reptation.

\section{Acknowledgments}

Fruitful discussions with A. Heuer and A. Kolb are gratefully acknowledged.

\section{References}

1. R. Graf, A. Heuer, and H. Spiess, Phys. Rev. Letters 80, 5738 (1998).

2. J. R. Havens and J. L. Koenig, J. Polym. Sci.: Polymer Letters Edition 21, 243 (1983).

3. G. D. Smith and D. Y. Yoon, J. Chem. Phys. 100, 649 (1994).

4. G. D. Smith, D. Y. Yoon, W. Zhu, and M. D. Ediger, Macromolecules 27, 5563 (1994).

5. N. E. Moe and M. D. Ediger, Polymer 37, 1787 (1996).

6. S. Trohalaki, Polymer 37, 1841 (1996).

7. A. Kolinski, J. Skolnick, and R. Yaris, Macromolecules 19, 2550 (1986).

8. D. Rigby and R.-J. Roe, J. Chem. Phys. 89, 5280 (1988).

9. K. Kremer and G. Grest, J. Chem. Phys. 92, 5057 (1990).

10. M. Pütz and A. Kolb, submitted to Comput. Phys. Commun., 1998. 